\title{
TU/e emonownen

\section{Manifestations of drag reduction by polymer additives in decaying, homogeneous, isotropic turbulence}

\section{Citation for published version (APA):}

Perlekar, P., Mitra, D., \& Pandit, R. (2006). Manifestations of drag reduction by polymer additives in decaying, homogeneous, isotropic turbulence. Physical Review Letters, 97(26), 264501-1/4.

https://doi.org/10.1103/PhysRevLett.97.264501

DOI:

10.1103/PhysRevLett.97.264501

Document status and date:

Published: 01/01/2006

\section{Document Version:}

Publisher's PDF, also known as Version of Record (includes final page, issue and volume numbers)

\section{Please check the document version of this publication:}

- A submitted manuscript is the version of the article upon submission and before peer-review. There can be important differences between the submitted version and the official published version of record. People interested in the research are advised to contact the author for the final version of the publication, or visit the $\mathrm{DOI}$ to the publisher's website.

- The final author version and the galley proof are versions of the publication after peer review.

- The final published version features the final layout of the paper including the volume, issue and page numbers.

Link to publication

\section{General rights}

Copyright and moral rights for the publications made accessible in the public portal are retained by the authors and/or other copyright owners and it is a condition of accessing publications that users recognise and abide by the legal requirements associated with these rights.

- Users may download and print one copy of any publication from the public portal for the purpose of private study or research.

- You may not further distribute the material or use it for any profit-making activity or commercial gain

- You may freely distribute the URL identifying the publication in the public portal.

If the publication is distributed under the terms of Article 25fa of the Dutch Copyright Act, indicated by the "Taverne" license above, please follow below link for the End User Agreement:

www.tue.nl/taverne

Take down policy

If you believe that this document breaches copyright please contact us at:

openaccess@tue.nl

providing details and we will investigate your claim. 


\title{
Manifestations of Drag Reduction by Polymer Additives in Decaying, Homogeneous, Isotropic Turbulence
}

\author{
Prasad Perlekar, ${ }^{1, *}$ Dhrubaditya Mitra, ${ }^{2, \dagger}$ and Rahul Pandit ${ }^{1, \$}$ \\ ${ }^{1}$ Centre for Condensed Matter Theory, Department of Physics, Indian Institute of Science, Bangalore 560012, India \\ ${ }^{2}$ Observatoire de la Côte d'Azur, B.P. 4229, 06304 Nice Cedex 4, France
}

(Received 28 September 2006; published 29 December 2006)

\begin{abstract}
The existence of drag reduction by polymer additives, well established for wall-bounded turbulent flows, is controversial in homogeneous, isotropic turbulence. To settle this controversy, we carry out a high-resolution direct numerical simulation of decaying, homogeneous, isotropic turbulence with polymer additives. Our study reveals clear manifestations of drag-reduction-type phenomena: On the addition of polymers to the turbulent fluid, we obtain a reduction in the energy-dissipation rate, a significant modification of the fluid energy spectrum especially in the deep-dissipation range, a suppression of small-scale intermittency, and a decrease in small-scale vorticity filaments.
\end{abstract}

The dramatic reduction of drag by the addition of small concentrations of polymers to a turbulent fluid continues to engage the attention of engineers and physicists. Significant advances have been made in understanding drag reduction both experimentally [1-3] and theoretically [4-7] in channel flows or the Kolmogorov flow [8]. However, the existence of drag-reduction-type phenomena in turbulent flows that are homogeneous and isotropic [919] remains controversial. Some experimental [15-18], numerical [11-14], and theoretical [9,10] studies have suggested that drag reduction should occur even in homogeneous, isotropic turbulence; but other studies have refuted this claim [19].

To settle this controversy, we have initiated an extensive direct numerical simulation (DNS) of decaying, homogeneous, isotropic turbulence in the presence of polymer additives. We monitor the decay of turbulence from initial states in which the kinetic energy of the fluid is concentrated at small wave vectors; this energy then cascades down to large wave vectors where it is dissipated by viscous effects; the energy-dissipation rate $\epsilon$ attains a maximum at $t_{m}$, roughly the time at which the cascade is completed. A recent shell-model study [13] has suggested that this peak in $\epsilon$ can be used to quantify drag reduction by polymer additives. Since shell models are far too simple to capture the complexities of real flows, we have studied decaying turbulence in the Navier-Stokes (NS) equation coupled to the finitely extensible nonlinear elastic Peterlin (FENE-P) model [20] for polymers. Our study, designed specifically to uncover drag-reduction-type phenomena, shows that the position of the maximum in $\epsilon$ depends only mildly on the polymer concentration $c$; however, the value of $\epsilon$ at this maximum falls as $c$ increases. We use this decrease of $\epsilon$ to define the percentage drag (or dissipation) reduction (DR) in decaying homogeneous, isotropic turbulence; we also explore other accompanying physical effects and show that they are in qualitative accord with drag- reduction experiments [18,21]: In particular, DR increases with $c$ (up to $25 \%$ in one of our simulations). For small values of $c$, the energy spectrum of the fluid is modified appreciably only in the dissipation range; however, this suffices to yield significant drag reduction. We show that vorticity filaments and intermittency are reduced at small spatial scales and that the extension of the polymers decreases as $c$ increases.

The NS and FENE-P (henceforth NSP) equations are

$$
\begin{gathered}
D_{t} \mathbf{u}=\nu \nabla^{2} \mathbf{u}+\frac{\mu}{\tau_{P}} \nabla \cdot\left[f\left(r_{P}\right) \mathcal{C}\right]-\nabla p ; \\
D_{t} \mathcal{C}=\mathcal{C} \cdot(\nabla \mathbf{u})+(\nabla \mathbf{u})^{T} \cdot \mathcal{C}-\frac{f\left(r_{P}\right) \mathcal{C}-I}{\tau_{P}} .
\end{gathered}
$$

Here $\mathbf{u}(\mathbf{x}, t)$ is the fluid velocity at point $\mathbf{x}$ and time $t$, incompressibility is enforced by $\nabla \cdot \mathbf{u}=0, D_{t}=\partial_{t}+\mathbf{u}$. $\nabla, \nu$ is the kinematic viscosity of the fluid, $\mu$ is the viscosity parameter for the solute (FENE-P), $\tau_{P}$ is the polymer relaxation time, $\rho$ is the solvent density (set to $1), p$ is the pressure, $(\nabla \mathbf{u})^{T}$ is the transpose of $(\nabla \mathbf{u}), \mathcal{C}_{\alpha \beta} \equiv$ $\left\langle R_{\alpha} R_{\beta}\right\rangle$ are the elements of the polymer-conformation tensor $\mathcal{C}$ (angular brackets indicate an average over polymer configurations), $I$ is the identity tensor with elements $\delta_{\alpha \beta}, f\left(r_{P}\right) \equiv\left(L^{2}-3\right) /\left(L^{2}-r_{P}^{2}\right)$ is the FENE-P potential that ensures finite extensibility, $r_{P} \equiv \sqrt{\operatorname{Tr}(\mathcal{C})}$ and $L$ are the length and the maximum possible extension, respectively, of the polymers, and $c \equiv \mu /(\nu+\mu)$ is a dimensionless measure of the polymer concentration [22]. $c=0.1$ corresponds, roughly, to $100 \mathrm{ppm}$ for polyethylene oxide [1].

We consider homogeneous, isotropic, turbulence, so we use periodic boundary conditions and solve Eq. (1) by using a massively parallel pseudospectral code [23] with $N^{3}$ collocation points in a cubic domain (side $\mathbb{L}=2 \pi$ ). We eliminate aliasing errors [23] by the $2 / 3$ rule, to obtain reliable data at small length scales, and use a second-order, slaved Adams-Bashforth scheme for time marching. For 
Eq. (2), we use an explicit sixth-order central-finitedifference scheme in space and a second-order AdamsBashforth method for temporal evolution. The numerical error in $r_{P}$ must be controlled by choosing a small time step $\delta t$; otherwise, $r_{P}$ can become larger than $L$, which leads to a numerical instability; this time step is much smaller than what is necessary for a pseudospectral DNS of the NS equation alone. Table I lists the parameters we use. We preserve the symmetric-positive-definite (SPD) nature of $\mathcal{C}$ at all times by using [22] the following Choleskydecomposition scheme: If we define $\mathcal{J} \equiv f\left(r_{P}\right) \mathcal{C}$, Eq. (2) becomes

$$
D_{t} \mathcal{J}=\mathcal{J} \cdot(\nabla \mathbf{u})+(\nabla \mathbf{u})^{T} \cdot \mathcal{J}-s(\mathcal{J}-\mathcal{I})+q \mathcal{J},
$$

where $s=\left(L^{2}-3+j^{2}\right) /\left(\tau_{P} L^{2}\right), \quad q=\left[d /\left(L^{2}-3\right)-\right.$ $\left.\left(L^{2}-3+j^{2}\right)\left(j^{2}-3\right) /\left(\tau_{P} L^{2}\left(L^{2}-3\right)\right)\right], j^{2} \equiv \operatorname{Tr}(\mathcal{J})$, and $d=\operatorname{Tr}\left[\mathcal{J} \cdot(\nabla \mathbf{u})+(\nabla \mathbf{u})^{T} \cdot \mathcal{J}\right]$. Since $\mathcal{C}$ and hence $\mathcal{J}$ are SPD matrices, we can write $\mathcal{J}=\mathcal{L} \mathcal{L}^{T}$, where $\mathcal{L}$ is a lower-triangular matrix with elements $\ell_{i j}$, such that $\ell_{i j}=$
TABLE I. The parameters $N, \delta t, L, \nu, \tau_{P}$, and $c$ for our four runs: NSP-96, NSP-192, NSP-256A, and NSP-256. NSP-96, NSP-192, and NSP-256A use type I initial conditions; NSP256 uses an initial condition of type II. We also carry out DNS studies of the NS equation with the same numerical resolutions as our NSP runs. Re $\equiv \sqrt{20} \mathcal{E}^{f, m} / \sqrt{3 \nu \epsilon^{f, m}}$ and $\mathrm{We} \equiv \tau_{P} \sqrt{\epsilon^{f, m} / \nu}$; NSP-96: $\mathrm{Re}=47.1$ and $\mathrm{We}=0.03,0.17,0.24,0.28,0.31,0.41$, $0.48,0.55,0.62,0.68,1.03$; NSP-192 and NSP-256A: $\mathrm{Re}=47.1$ and $\mathrm{We}=0.35 ; \mathrm{NSP}-256: \mathrm{Re}=126.6$ and $\mathrm{We}=0.76$.

\begin{tabular}{lcccccc}
\hline \hline & $N$ & $\delta t$ & $L$ & $\nu$ & $\tau_{P}$ & $c$ \\
\hline NSP-96 & 96 & $1.0 \times 10^{-2}$ & 100 & $10^{-2}$ & $0.1-3$ & $0.1,0.2,0.3,0.4$ \\
NSP-192 & 192 & $1.0 \times 10^{-2}$ & 100 & $10^{-2}$ & 1 & $0.1,0.4$ \\
NSP-256A & 256 & $1.0 \times 10^{-2}$ & 100 & $10^{-2}$ & 1 & $0.1,0.4$ \\
NSP-256 & 256 & $4.0 \times 10^{-3}$ & 100 & $10^{-3}$ & 1 & $0.1,0.4$ \\
\hline \hline
\end{tabular}

0 for $j>i$. Thus, Eq. (3) now yields $\left(1 \leq i \leq 3\right.$ and $\Gamma_{i j}=$ $\left.\partial_{i} u_{j}\right)$

$$
\begin{aligned}
& D_{t} \ell_{i 1}=\sum_{k} \Gamma_{k i} \ell_{k 1}+\frac{1}{2}\left[(q-s) \ell_{i 1}+(-1)^{(i \bmod 1)} \frac{s \ell_{i 1}}{\ell_{11}^{2}}\right]+\left(\delta_{i 3}+\delta_{i 2}\right) \frac{\ell_{i 2}}{\ell_{11}} \sum_{m>1} \Gamma_{m 1} \ell_{m 2}+\delta_{i 3} \Gamma_{i 1} \frac{\ell_{33}^{2}}{\ell_{11}}, \quad \text { for } i \geq 1 ; \\
& D_{t} \ell_{i 2}=\sum_{m \geq 2} \Gamma_{m i} \ell_{m 2}-\frac{\ell_{i 1}}{\ell_{11}} \sum_{m \geq 2} \Gamma_{m 1} \ell_{m 2}+\frac{1}{2}\left[(q-s) \ell_{i 2}+(-1)^{(i+2)} s \frac{\ell_{i 2}}{\ell_{22}^{2}}\left(1+\frac{\ell_{21}^{2}}{\ell_{11}^{2}}\right)\right]+\delta_{i 3}\left[\frac{\ell_{33}^{2}}{\ell_{22}}\left(\Gamma_{32}-\Gamma_{31} \frac{\ell_{21}}{\ell_{11}}\right)+s \frac{\ell_{21} \ell_{31}}{\ell_{11}^{2} \ell_{22}}\right], \\
& \text { for } i \geq 2 ; \\
& D_{t} \ell_{33}=\Gamma_{33} \ell_{33}-\ell_{33}\left[\sum_{m<3} \frac{\Gamma_{3 m} \ell_{3 m}}{\ell_{m m}}\right]+\frac{\Gamma_{31} \ell_{32} \ell_{21} \ell_{33}}{\ell_{11} \ell_{22}}-s \frac{\ell_{21} \ell_{31} \ell_{32}}{\ell_{11}^{2} \ell_{22} \ell_{33}}+\frac{1}{2}\left[(q-s) \ell_{33}+\frac{s}{\ell_{33}}\left(1+\sum_{m<3} \frac{\ell_{3 m}^{2}}{\ell_{m m}^{2}}\right)+\frac{s \ell_{21}^{2} \ell_{32}^{2}}{\ell_{11}^{2} \ell_{22}^{2} \ell_{33}}\right] .
\end{aligned}
$$

The SPD nature of $\mathcal{C}$ is preserved by Eq. (4) if $\ell_{i i}>0$, which we enforce explicitly [22] by considering the evolution of $\ln \left(\ell_{i i}\right)$ instead of $\ell_{i i}$.

We use the following initial conditions (superscript 0): $\mathcal{C}_{m n}^{0}(\mathbf{x})=\delta_{m n}$ for all $\mathbf{x}$; and $u_{m}^{0}(\mathbf{k})=P_{m n}(\mathbf{k}) v_{n}^{0}(\mathbf{k}) \times$ $\exp \left(i \theta_{n}(\mathbf{k})\right)$, with $m, n=x, y, z, P_{m n}=\left(\delta_{m n}-k_{m} k_{n} / k^{2}\right)$ the transverse projection operator, $\mathbf{k}$ the wave vector with components $k_{m}=(-N / 2,-N / 2+1, \ldots, N / 2), k=|\mathbf{k}|$, $\theta_{n}(\mathbf{k})$ random numbers distributed uniformly between 0 and $2 \pi$, and $v_{n}^{0}(\mathbf{k})$ chosen such that the initial kineticenergy spectra are either of type I, with $E^{\mathrm{I}}(k)=$ $k^{2} \exp \left(-2 k^{4}\right)$, or of type II, with $E^{\mathrm{II}}(k)=k^{4} \exp \left(-2 k^{2}\right)$.

In addition to $\mathbf{u}(\mathbf{x}, t)$, its Fourier transform $\mathbf{u}_{\mathbf{k}}(t)$, and $\mathcal{C}(\mathbf{x}, t)$, we monitor the vorticity $\omega \equiv \nabla \times \mathbf{u}$, the kineticenergy spectrum $E(k, t) \equiv \sum_{k-1 / 2<k^{\prime} \leq k+1 / 2}\left|\mathbf{u}_{\mathbf{k}^{\prime}}^{2}(t)\right|$, the total kinetic energy $\mathcal{E}(t) \equiv \sum_{k} E(k, t)$, the energy-dissipation rate $\epsilon(t) \equiv \nu \sum_{k} k^{2} E(k, t)$, the cumulative probability distribution of scaled polymer extensions $P^{C}\left(r_{P}^{2} / L^{2}\right)$, and the hyperflatness $\quad \mathcal{F}_{6}(r) \equiv \mathcal{S}_{6}(r) / \mathcal{S}_{2}^{3}(r)$, where $\quad \mathcal{S}_{p}(r) \equiv$ $\left\langle\{[\mathbf{u}(\mathbf{x}+\mathbf{r})-\mathbf{u}(\mathbf{x})] \cdot \mathbf{r} / r\}^{p}\right\rangle$ is the order- $p$ longitudinal velocity structure function and the angular brackets denote an average over our simulation domain at $t_{m}$. For notational convenience, we do not display the dependence on $c$ explicitly.
Figure 1(a) shows that $\epsilon$ first increases with time, reaches a peak, and then decreases; for $c=0$, this peak occurs at $t=t_{m}$. The position of this peak changes mildly with $c$, but its height goes down significantly as $c$ increases. This suggests the following natural definition [13] of the percentage drag or dissipation reduction for decaying homogeneous, isotropic turbulence:

$$
\mathrm{DR} \equiv\left(\frac{\boldsymbol{\epsilon}^{f, m}-\boldsymbol{\epsilon}^{p, m}}{\boldsymbol{\epsilon}^{f, m}}\right) \times 100 ;
$$

here (and henceforth) the superscripts $f$ and $p$ stand, respectively, for the fluid without and with polymers, and the superscript $m$ indicates the time $t_{m}$. Figure 1(b) shows plots of DR versus $c$, for the Weissenberg number We $\equiv$ $\tau_{P} \sqrt{\epsilon^{f, m} / \nu} \simeq 0.35$, and versus $\mathrm{We}$, for $c=1 / 11 \simeq 0.1$. DR increases with $c$ in qualitative accord with experiments on channel flows (where DR is defined via a normalized pressure difference); but it drops gently as We increases, in contrast to the behavior seen in channel flows (in which $\tau_{P}$ is varied by changing the polymer).

In decaying turbulence, the total kinetic energy $\mathcal{E}(t)$ of the fluid falls as $t$ increases; the rate at which it falls increases with $c$ [Fig. 1(c)], which suggests that the addi- 

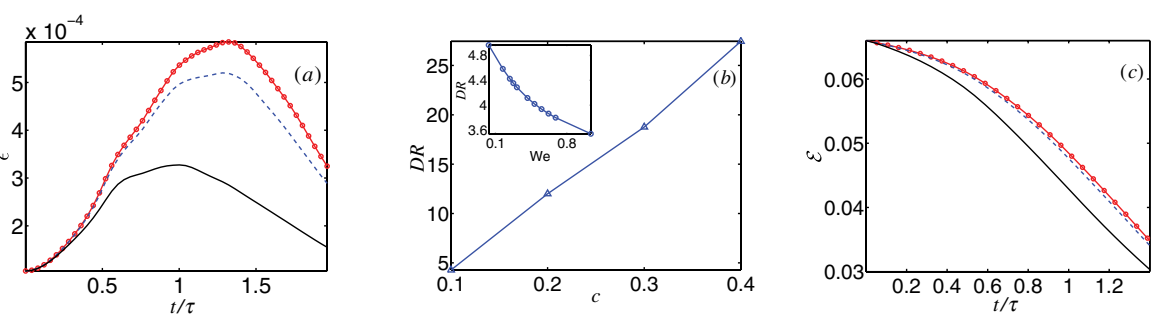

FIG. 1 (color online). (a) Temporal evolution of the energy-dissipation rate $\epsilon$ (run NSP-256) for concentrations $c=0.1$ (dashed blue line) and $c=0.4$ (solid line), with $\tau \equiv \sqrt{2 \mathcal{E}(t=0) / 3 \llbracket^{2}}$; (b) percentage DR versus $c$ (run NSP-192); the inset shows the mild variation in DR with We (run NSP-96); (c) temporal evolution of the total fluid energy $\mathcal{E}$ for concentrations $c=0.1$ (dashed blue line) and $c=0.4$ (solid line) (run NSP-256). In (a) and (c), the plots for $c=0$ (dotted red line) are shown for comparison.

tion of polymers increases the effective viscosity of the solution. This is not at odds with the decrease of $\epsilon$ with increasing $c$, since the effective viscosity because of polymers turns out to be scale-dependent. We confirm this by obtaining the kinetic-energy spectrum $E^{p, m}(k)$ for the fluid in the presence of polymers at $t=t_{m}$. For small concentrations $(c \simeq 0.1)$, the spectra with and without polymers differ substantially only in the deep-dissipation range, where $E^{f, m}(k) \ll E^{p, m}(k)$. As $c$ increases, say, $c \simeq 0.4$, $E^{p, m}(k)$ is reduced relative to $E^{f, m}(k)$ at intermediate values of $k$ [Fig. 2(a)]; however, deep in the dissipation range $E^{f, m}(k) \ll E^{p, m}(k)$. We now define [12] the effective scale-dependent viscosity $\nu_{e}(k) \equiv$ $\nu+\Delta \nu(k)$, with $\Delta \nu(k) \equiv-\mu \sum_{k-1 / 2<k^{\prime} \leq k+1 / 2} \mathbf{u}_{\mathbf{k}^{\prime}} \cdot(\nabla \cdot$ $\mathcal{J})_{-\mathbf{k}^{\prime}} /\left[\tau_{P} k^{\prime 2} E^{p, m}\left(k^{\prime}\right)\right]$, where $(\nabla \cdot \mathcal{J})_{\mathbf{k}}$ is the Fourier transform of $\nabla \cdot \mathcal{J}$. The inset in Fig. 2(a) shows that $\Delta \nu(k)>0$ for $k<15$, but $\Delta \nu(k)<0$ around $k=20$. This explains why $E^{p, m}(k)$ is suppressed relative to $E^{f, m}(k)$ at small $k$, rises above it in the deep-dissipation range, and crosses over from its small- $k$ to large- $k$ behaviors around the value of $k$ where $\Delta \nu(k)$ goes through zero.

Given the resolution of our DNS, inertial-range intermittency can be studied only by using extended selfsimilarity [24] as we will report elsewhere. However, we explore dissipation-range statistics further by calculating the hyperflatness $\mathcal{F}_{6}(r)$ [Fig. 2(b)]. The addition of polymers slows down the growth of $\mathcal{F}_{6}(r)$, as $r \rightarrow 0$, which signals the reduction of small-scale intermittency. This is further supported by the iso- $|\omega|$ surfaces shown in Fig. 3. If no polymers are present, these iso- $|\omega|$ surfaces are filamentary [25] for large $|\omega|$; polymers suppress a significant fraction of these filaments.

We use a rank-order method [26] to obtain $P^{C}\left(r_{P}^{2} / L^{2}\right)$ and find that, as $c$ increases [Fig. 2(c)], the extension of the polymers decreases. We have checked that, in the passivepolymer version of Eqs. (1) and (2), the extension of polymers is much more than in Fig. 2(c).

Our study contrasts clearly drag reduction in homogeneous, isotropic, turbulence and in wall-bounded flows. In both of these cases, the polymers increase the overall viscosity of the solution (see, e.g., Fig. 1(c) and Ref. [12]). In wall-bounded flows, the presence of polymers inhibits the flow of the streamwise component of the momentum into the wall, which, in turn, increases the net throughput of the fluid and thus results in drag reduction, a mechanism that can have no analog in homogeneous, isotropic turbulence. However, the decrease of $\epsilon(t)$ with increasing $c$ [Fig. 1(b)] yields a natural definition of DR [Eq. (5)] for this case [27]. Thus, if the term drag reduction must be reserved for wall-bounded flows, then we suggest the expression dissipation reduction for homogeneous, isotropic turbulence. We have shown that $\nu_{e}$ must be scale-dependent; its counterpart in wall-bounded flows is the position-dependent viscosity of Refs. [4,7]. Furthermore, as in wall-bounded flows, an increase in $c$ leads to an increase in DR [Fig. 1(b)]. In channel flows, an
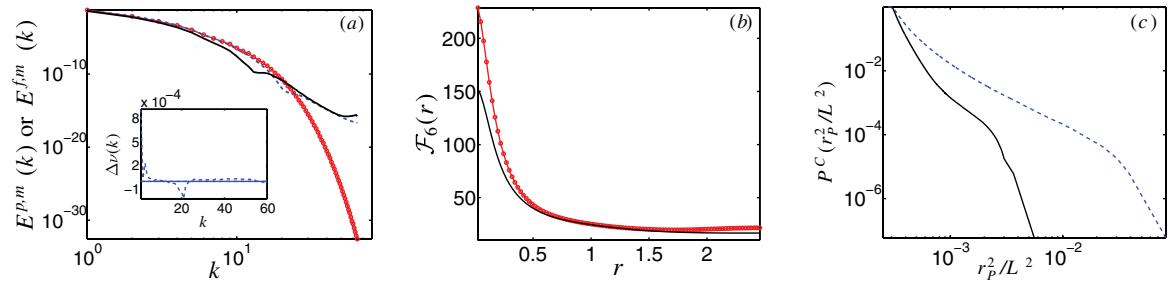

FIG. 2 (color online). (a) Plots of the energy spectra $E^{p, m}(k)$ or $E^{f, m}(k)$ versus $k$ (run NSP-192) for $c=0.1$ (dashed blue line) and $c=0.4$ (solid line) $\left[E^{p, m}(k)\right.$ is unchanged if we use $N=256$, with all other parameters the same (run NSP-256A)]; inset: polymer contribution to the scale-dependent viscosity $\Delta \nu(k)$ versus $k$ for $c=0.1$ (dashed blue line); $\Delta \nu(k)=0$ (solid line) is also shown for reference; (b) the hyperflatness $\mathcal{F}_{6}(r)$ as a function of $r$ (run NSP-256) and concentration $c=0.4$ (solid line). In (a) and (b), the corresponding plots with $c=0$ (dotted red line) are shown for comparison. (c) The cumulative probability distribution function $P^{C}\left(r_{P}^{2} / L^{2}\right)$ versus $r_{P}^{2} / L^{2}$ for $c=0.1$ (dashed blue line) and $c=0.4$ (solid line) (run NSP-256). 

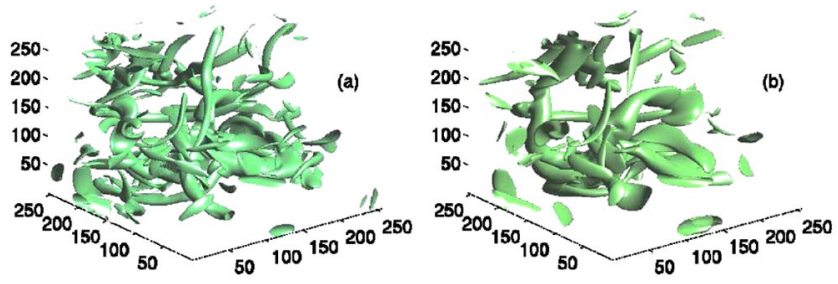

FIG. 3 (color online). Constant- $|\omega|$ isosurfaces for $|\omega|=$ $\langle|\omega|\rangle+2 \sigma$ at $t_{m}$ (a) without and (b) with polymers (run NSP256), and $c=0.4 ;\langle|\omega|\rangle$ is the mean and $\sigma$ the standard deviation of $|\omega|$.

increase in We leads to an increase in DR, but we find that DR falls marginally as We increases [Fig. 1(b)].

Our DNS of the Navier-Stokes equation with polymer additives [Eqs. (1) and (2)] resolves the controversy about drag reduction in decaying homogeneous, isotropic turbulence and shows clearly that Eq. (5) offers a natural definition of DR for this case in a far more realistic model than those of Refs. [11,13]. We also find a nontrivial modification of the fluid kinetic-energy spectrum especially in the deep-dissipation range [Fig. 2(b)] that can be explained in terms of a polymer-induced, scale-dependent viscosity. Experiments [16,17] do not resolve the dissipation range as clearly as we do, so the experimental verification of the deep-dissipation-range behavior of Fig. 2(a) remains a challenge. Earlier theoretical studies [10,11] have also not concentrated on this dissipation range. The reduction in the small-scale intermittency [Fig. 2(b)] and in the constant- $|\omega|$ isosurfaces [Fig. 3] is in qualitative agreement with channel-flow studies [2], where a decrease in the turbulent volume fraction is seen on the addition of the polymers, and water-jet studies [21], where the addition of the polymers leads to a decrease in small-scale structures. We hope our work will stimulate more experimental studies of drag or dissipation reduction in homogeneous, isotropic turbulence.

We thank C. Kalelkar, R. Govindarajan, V. Kumar, S. Ramaswamy, L. Collins, and A. Celani for discussions, CSIR, DST, and UGC (India) for financial support, and SERC (IISc) for computational facilities.

*Electronic address: perlekar@physics.iisc.ernet.in †Electronic address: Dhrubaditya.MITRA@obs-nice.fr

¥Also at Jawaharlal Nehru Centre for Advanced Scientific Research, Jakkur, Bangalore, India.

Electronic address: rahul@physics.iisc.ernet.in

[1] P. Virk, AIChE J. 21, 625 (1975).

[2] P. van Dam, G. Wegdam, and J. van der Elsken, J. NonNewtonian Fluid Mech. 53, 215 (1994).
[3] J. D. Toonder, M. Hulsen, G. Kuiken, and F. Nieuwstadt, J. Fluid Mech. 337, 193 (1997).

[4] J. Lumley, J. Polym. Sci. Macromol. Rev. 7, 263 (1973).

[5] K. Sreenivasan and C. White, J. Fluid Mech. 409, 149 (2000).

[6] P. Ptasinski et al., J. Fluid Mech. 490, 251 (2003).

[7] V. L'vov, A. Pomyalov, I. Procaccia, and V. Tiberkevich, Phys. Rev. Lett. 92, 244503 (2004).

[8] G. Boffetta, A. Celani, and A. Mazzino, Phys. Rev. E 71, 036307 (2005).

[9] M. Tabor and P. G. de Gennes, Europhys. Lett. 2, 519 (1986).

[10] J. K. Bhattacharjee and D. Thirumalai, Phys. Rev. Lett. 67, 196 (1991).

[11] R. Benzi, E. de Angelis, R. Govindarajan, and I. Procaccia, Phys. Rev. E 68, 016308 (2003).

[12] R. Benzi, E. Ching, and I. Procaccia, Phys. Rev. E 70, 026304 (2004) consider a scale-dependent viscosity for a shell model (but use an artificial diffusivity for polymers for numerical stability).

[13] C. Kalelkar, R. Govindarajan, and R. Pandit, Phys. Rev. E 72, 017301 (2005).

[14] E. de Angelis, C. Casicola, R. Benzi, and R. Piva, J. Fluid Mech. 531, 1 (2005).

[15] E. van Doorn, C. White, and K. Sreenivasan, Phys. Fluids 11, 2387 (1999).

[16] W. McComb, J. Allan, and C. Greated, Phys. Fluids 20, 873 (1977).

[17] C. Friehe and W. Schwarz, J. Fluid Mech. 44, 173 (1970).

[18] D. Bonn, Y. Couder, P. van Dam, and S. Douady, Phys. Rev. E 47, R28 (1993).

[19] D. Bonn et al., J. Phys. Condens. Matter 17, S1219 (2005).

[20] A. Peterlin, J. Polym. Sci., Part B: Polym. Lett. 4, 287 (1966); H. Warner, Ind. Eng. Chem. Fundam. 11, 379 (1972);

R. Armstrong, J. Chem. Phys. 60, 724 (1974); E. Hinch, Phys. Fluids 20, S22 (1977).

[21] J. Hoyt and J. Taylor, Phys. Fluids 20, S253 (1977).

[22] T. Vaithianathan and L. Collins, J. Comput. Phys. 187, 1 (2003). We correct their Eq. (40) and definition of $q$.

[23] A. Vincent and M. Meneguzzi, J. Fluid Mech. 225, 1 (1991); C. Canuto, M. Hussaini, A. Quarteroni, and T. Zang, Spectral Methods in Fluid Dynamics (SpringerVerlag, Berlin, 1988).

[24] R. Benzi et al., Phys. Rev. E 48, R29 (1993); S. Dhar, A. Sain, and R. Pandit, Phys. Rev. Lett. 78, 2964 (1997).

[25] Y. Kaneda et al., Phys. Fluids 15, L21 (2003).

[26] D. Mitra, J. Bec, R. Pandit, and U. Frisch, Phys. Rev. Lett. 94, 194501 (2005).

[27] In some steady-state simulations [11,22], DR is associated with $E^{p}(k)>E^{f}(k)$, for small $k$. We obtain this for type II, but not type I, initial conditions; but Eq. (5) yields drag reduction for both of these initial conditions. 